\title{
Electron beam-induced mass transport in As-Se thin films: compositional dependence and glass network topological effects
}

\author{
M L Trunov ${ }^{1}$, C Cserháti ${ }^{2}$, P M Lytvyn ${ }^{3}$, Yu Kaganovskii ${ }^{4}$ and \\ S Kökényesi ${ }^{5}$ \\ ${ }^{1}$ Uzhgorod National University, Pidhirna Street, 46 Uzhgorod 88000, Ukraine \\ ${ }^{2}$ Department of Solid State Physics, University of Debrecen, H-4010 Debrecen, Hungary \\ ${ }^{3}$ Institute of Semiconductor Physics, NAS of Ukraine, Kiev 03028, Ukraine \\ ${ }^{4}$ Department of Physics, Bar-Ilan University, Ramat-Gan 52900, Israel \\ ${ }^{5}$ Department of Experimental Physics, University of Debrecen, H-4010 Debrecen, Hungary \\ E-mail: trunov.m@gmail.com (M L Trunov)
}

Received 25 November 2012, in final form 3 April 2013

Published

Online at stacks.iop.org/JPhysD/46

\begin{abstract}
Electron beam (e-beam)-induced changes of surface profile morphology in $\mathrm{As}_{c} \mathrm{Se}_{1-c}$ $(0.2<c<0.5)$ thin films are investigated as a function of the film composition. It is shown that the extent and value of local surface alterations follow the composition-related changes of glass parameters such as softening temperature and glass network connectivity. The giant e-beam-induced surface relief changes detected in the films $\mathrm{As}_{0.2} \mathrm{Se}_{0.8}$ are connected with lateral mass transport, which increases drastically near rigidity transition, i.e. at a coordination number $r \sim 2.2$ of the glass structures when the rigidity starts to percolate through the structure. The model of the process, which reflects the compositional dependence of the stimulated mass transport, is presented.
\end{abstract}

(Some figures may appear in colour only in the online journal)

\section{Introduction}

Over the years, research on chalcogenide glasses $(\mathrm{ChG})$ i.e. the materials, which include atoms of selenium, sulfur or tellurium, has grown enormously due to the strong interest in these materials from both the scientific and technological point of view [1-3]. Applications of ChGs have mainly been based on their transparency to infrared light (passive) and their sensitivity to different kind of irradiation (active). The latter produce pronounced structural changes that are scientifically the more important case, regarding to the challenges of understanding their microscopic nature and applications as functional materials of photonics.

As the external stimuli, which produce noticeable structural rearrangement in the irradiated volume, the photon, $\gamma$, electron (e-beam) or ion irradiation are widely used [4-7]. During and after irradiation, mechanical, optical, rheological and other physical and chemical properties of ChG undergo alterations. It should be noted that these changes are partially or even completely reversible and initial state can be restored by heating to the glass transition temperature. These effects could be used in different applications, including direct fabrication of optical elements (lenses and waveguides) and low-cost memory devices (optical discs).

From the general point of view, the basic effects of irradiation on $\mathrm{ChG}$ are very similar since the well-known irradiation-induced stimulated transformations occur at the initial level of electron-hole excitation and further structural transformations, causing the same physical and/or chemical changes including optical (darkening and optical anisotropy), mechanical (softening), geometrical effects (expansion or contraction) and so on. Among them, changes in rheological properties including viscous flow under external load, i.e. fluidity, termed as irradiation-induced plastic effects [8], 
are of high importance posing challenges for both the understanding of the structural changes involved and the possible technological applications.

A special case of those effects, i.e. direct light-induced fabrication of surface relief (SR) by lateral mass transport on ChG films, have been widely studied for several selected composition, including $\mathrm{As}_{0.4} \mathrm{~S}_{0.6}, \mathrm{As}_{0.2} \mathrm{Se}_{0.8}, \mathrm{Se}$ [9-12] and appropriate mechanisms were proposed recently $[13,14]$. More detailed study reveals the influence of chalcogen content on this process. In the previous papers [10], the authors investigated the light-induced $\mathrm{SR}$ formation in $\mathrm{As}_{c} \mathrm{Se}_{1-c}$ by lateral mass transport and elucidated that $\mathrm{ChG}$ films of $\mathrm{As}_{0.2} \mathrm{Se}_{0.8}$ composition are the most efficient in this system for SR formation, with large peak-to-valley amplitude produced by lateral mass transport under projection of a p-polarized interference pattern.

Electron beam (e-beam)-induced surface patterning of ChG has attracted considerably less attention and successful attempts to obtain SR on ChG thin films till now have been made on the basis of well-known two-step method exploiting the phenomenon of photo-induced changes in the dissolution rate of $\mathrm{ChG}$ (see reviews $[15,16]$ as an example). A limited number of thorough experimental studies devoted to the direct formation of SR by e-beam exposure have been performed recently. These studies mainly have been focused on the selected homogeneous (stoichiometric) compositions like $\mathrm{Ge}_{0.2} \mathrm{Se}_{0.8}, \mathrm{As}_{0.4} \mathrm{~S}_{0.6}, \mathrm{As}_{0.4} \mathrm{Se}_{0.6}$ films [17-24] or nanostructured chalcogenide layers [25, 26].

The composition dependence of various physical properties and their changes under different kind of irradiation is an important aspect of the study of chalcogenide network glasses and films. There is not much information available about the response of amorphous thin films with different compositions to the e-beam. We have found only the paper devoted to such a response for thin films of $\mathrm{As}_{c} \mathrm{Se}_{1-c}$ system mostly for stoichiometric and over-stoichiometric compositions $(0.4 \leqslant c \leqslant 0.7)$ [18]. Summarizing, such type of experiments revealed that different kind of surface deformation (ridges or grooves) takes place by e-beam irradiation depending on the composition and the electron current dose. Concerning the mechanism of these effects, a quantitative evaluation of the surface deformation has suggested that this phenomenon appears through two processes, the electrostatic field that arises inside the film and electron-induced fluidity [19].

Recently, we reported the influence of the e-beam on surface stability of non-annealed $\mathrm{As}_{0.2} \mathrm{Se}_{0.8}$ film and found that formation of ridges and depressions near the ridges were induced via the e-beam accelerated lateral mass transport [27]. The aim of this work is to extend our previous results to surface e-beam-induced patterning of amorphous films of $\mathrm{As}_{c} \mathrm{Se}_{1-c}$ system, for $0.2<c \leqslant 0.5$. We chose this system for the following reasons: (i) it allows the synthesis of stable glasses over a wide range of compositions including Se-enriched glasses and possesses reproducible physical properties; (ii) there is no information available about the response of amorphous As-Se thin films with different compositions for e-beam irradiation, especially in $c \leqslant 0.3$ range; (iii) these materials are suitable as an object for modelling of the network glass structure that shows unusual rigidity percolation in the glass structure at mean coordination number $r=2.29$. This value is significantly lower than the mean-field rigidity percolation transition value of 2.40 for the observed onset of rigidity in most well-known system of ChG [28] ( $r$ is defined as the average number of covalent bonds per atom [29]). With the generally accepted point of view that the largescale response of $\mathrm{ChG}$ to the external perturbation should be expected for under-constrained floppy system (see [30]), this rigidity transformation promises maximum in the trend of ebeam-induced relief formation in Se-reach glasses. In such a case, the contribution of $\mathrm{ChG}$ structure to the e-beam response could be revealed and discussed.

\section{Experimental}

Bulk glasses of $\mathrm{As}_{c} \mathrm{Se}_{1-c}$ (where $c=0.2,0.3,0.4$ and 0.5) were synthesized first from the pure (99.999) elements in evacuated quartz tubes, followed by quenching of the melt. Amorphous films with thicknesses ranging from $800 \mathrm{~nm}$ to several micrometres were produced by vacuum thermal deposition at a rate of $3-6 \mathrm{~nm} \mathrm{~s}^{-1}$ onto an unheated glass substrates covered by conductive ITO sublayer (to avoid the effect of charging during e-beam exposure). Note that Au layer evaporated onto chalcogenide surface could be used instead of ITO sublayer as a conductive agent. Detailed experiments with thin Au overlayer (10-30 nm in thickness) show, however, that serious distortion of the surface up to crack formation takes place very often and causes surface instability and bad reproducibility of the collected data.

The film thicknesses $d$ were measured in situ during deposition interferometrically and the chemical composition and homogeneity of the films were monitored through appropriate energy dispersive $\mathrm{X}$-ray analysis (EDAX) measurements after the deposition. Selected films were annealed at the corresponding glass transition temperature for $1 \mathrm{~h}$ at ambient conditions. To ensure the similarity of the results for all the data presented in this article, the thickness of the film was $2 \mu \mathrm{m}$. The chemical composition and homogeneity of the films were monitored through EDAX and Raman scattering before and after e-beam irradiation. Appropriate analysis was made and no noticeable differences were found in the compositions.

Recording of lines $\sim 40 \mu \mathrm{m}$ length was made in Hitachi S4300 cold field emission (CFE) scanning electron microscope (SEM) at different beam energies (10-20 keV) and currents (5-10 nA). The beam current was measured prior to and after writing, using a Faraday cup in the SEM chamber. The part of clean surface of the film with $40 \mu \mathrm{m}$ writing length was exposed by e-beam focused to $50-100 \mathrm{~nm}$ spot with a forth (on)-and-back(off) writing scheme, which has been scanned along a line (the line dose was $3.5 \times 10^{-2} \mu \mathrm{C} \mathrm{cm}^{-1}$ ). The times of exposure were varied from 10 to $300 \mathrm{~s}$ for each selected composition.

The surface of amorphous $\mathrm{As}_{c} \mathrm{Se}_{1-c}$ layers and the features of final patterns were analysed after e-beam drawing using a Nanoscope Dimension 3100 atomic force microscope 


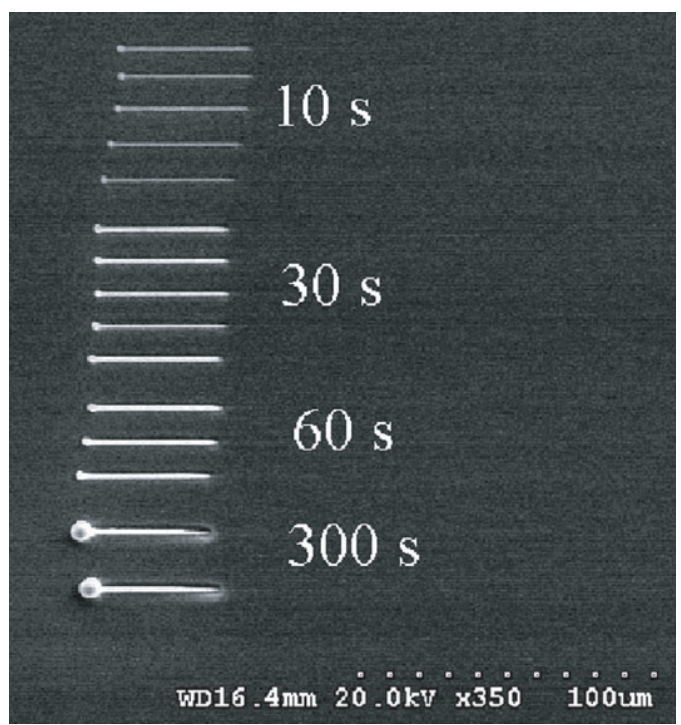

Figure 1. SEM view of e-beam-induced lines with different exposure time $(U=20 \mathrm{kV}, I=7 \mathrm{nA})$.

(AFM) (VEECO), which operated in a tapping mode. The surface profiles were calculated from the appropriate crosssections and reported values were the results of averaging from 2 to 5 measurements depending on the exposure. Reproducibility of the results can be seen in SEM pictures (figure 1). Each line was produced by a circular e-beam (at the accelerating voltage of $20 \mathrm{kV}$ ), which scanned along the line from left to right. The deflecting voltage had a saw-tooth shape with $4 \mathrm{~ms}$ delay between the $16 \mathrm{~ms}$ ramp and the falling edge of the signal. As the e-beam waited $4 \mathrm{~ms}$ at the beginning of each line before it started scanning, time of e-beam interaction with the film in this point exceeded several times the average interaction time at the line. This causes a spherical shape of the hillock at the edge.

It should be mentioned here that experiments on e-beam recording were extended to compositions with $c<0.2$ too, up to the pure Se, and the recording efficiency (relief height) was not less as for $c=0.2$, but the stability of the layers decreases, especially in pure Se due to its low glass-transition temperature of $\sim 30^{\circ} \mathrm{C}$ [31]. E-beam recording in the materials with $0 \leqslant c<0.2$ are still under study and the results will be described in a separate paper.

\section{Results}

The colour of the deposited films varied from light to dark red depending on the Se concentration. Preliminary tests, not involving e-beam irradiation, were performed on the asdeposited and annealed chalcogenide films to assess their amorphous nature and their surface morphology. The films showed smooth surfaces on AFM images: the surface of amorphous films was irregular with a typical roughness of 2-5 nm, which may correspond to the scale of medium-range structures.

Films of all compositions gave rise to surface patterning under e-beam irradiation, as it is shown in figure $2(a)$, as an example. Similarly to [19], we have found that the irradiated regions look as the ridges and depressions on the periphery (figure 2(b)). For quantitative estimation, we have measured the height of the lines from the bottom of the depressions near the ridges to the top of the central peak, see figure $2(b)$ as an example. In this experiment, the material moves towards e-beam, and the volume of depressions is close to the volume of ridges.

The e-beam-induced formation of ridges is revealed for all investigated compositions irrespective to the time of exposure. Note that both the ridges and depressions near the ridges grow with the exposure time. It means that the relief is induced by e-beam-accelerated lateral mass transport. In figures 3(a)$(h)$, we have summarized the results of relief formation in As-Se films examined by AFM immediately after the e-beam treatment.

We also noticed that there was no significant change in the height or shape of any of the lines after storing the samples in air at ambient conditions for six months.

Figures 3(a)- $(f)$ reveal typical AFM images of e-beaminduced reliefs for selected compositions of As-Se films, both as-prepared and annealed. Figure 3( $g$ ) shows appropriate cross-sections of the profiles for a given e-beam exposure. The relief formations are observed to be strong functions of the compositions and thermal prehistory of the samples (asprepared or annealed) and exposure. A quantitative correlation between the above-mentioned parameters and feature height is shown in figure $3(h)$. The relief formation versus exposure for $\mathrm{As}_{0.2} \mathrm{Se}_{0.8}$ films (both as-prepared and annealed) was also undertaken and additional details of the process were discovered (see figure 4). The results showed that prolonged ebeam exposure triggers a giant mass transport and accelerates SR formation while increasing it in height up to the order of the film thickness, figures 4(a) and (b). Appreciable changes in the SR kinetics with prolonged exposure take place. Figure $4(c)$ shows the two-stage process of SR formation under e-beam exposure in as-deposited (curve 1) and annealed (curve 2) $\mathrm{As}_{0.2} \mathrm{Se}_{0.8}$ films. A small SR immediately appears upon e-beam irradiation whose height reaches a value of about $10-30 \mathrm{~nm}$ (depending on thermal prehistory of the film) even if the exposure time is as short as $10 \mathrm{~s}$. The SR height remains unchanged after ceasing irradiation. With further e-beam exposure, we observe a linear growth of SR height. Note that the process of the linear growth in SR disappears completely in As-rich films, i.e. for $\mathrm{As}_{0.4} \mathrm{Se}_{0.6}$ and $\mathrm{As}_{0.5} \mathrm{Se}_{0.5}$ compositions (the appropriate images are not presented here).

Some features obtained are worth to be mentioned and summarized.

First, the height of the e-beam-induced lines depends almost linearly on the composition for $0.2 \leqslant c \leqslant 0.4$ and changes much less for $0.4 \leqslant c \leqslant 0.5$. According to the figures 3 and 4, the best conditions for the e-beam recording in thin films of As-Se system are near the composition with $c=0.2$. At the same time, we have found only slight surface response for as-deposited film of stoichiometric $\left(\mathrm{As}_{0.4} \mathrm{Se}_{0.6}\right)$ and As-enriched $\left(\mathrm{As}_{0.5} \mathrm{Se}_{0.5}\right)$ compositions at exposure from $10 \mathrm{~s}$ to $30 \mathrm{~s}$, respectively. According to the AFM images and appropriate cross-sections, the surface perturbation looks as very small distortions, several nanometres in height, see 

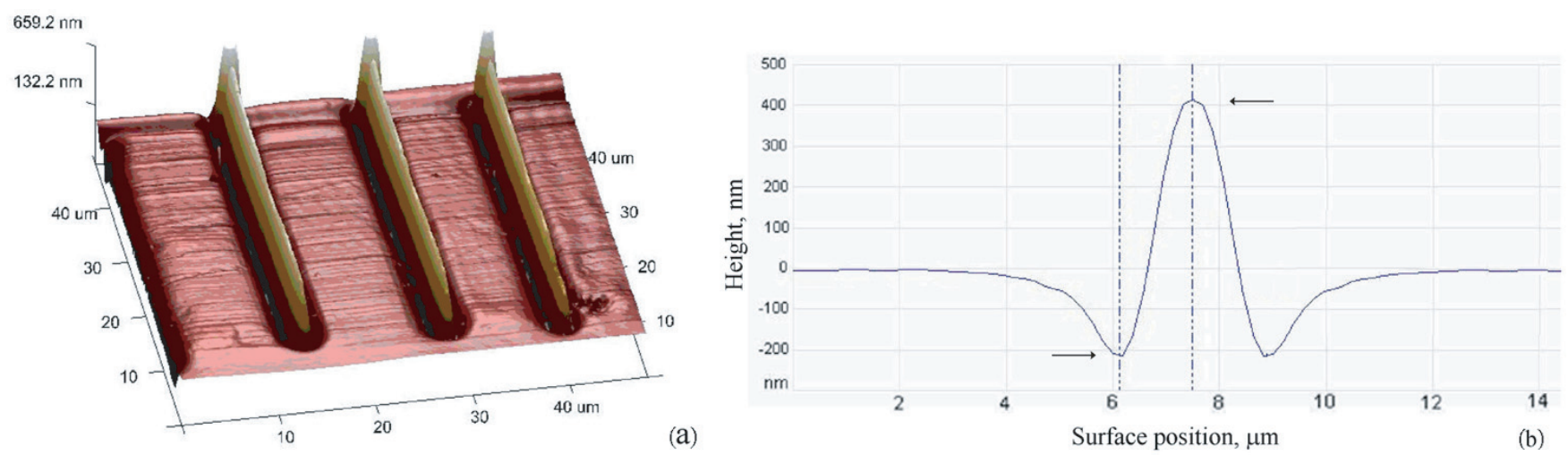

Figure 2. Lines produced by e-beam for $\mathrm{As}_{0.2} \mathrm{Se}_{0.8}$ composition: (a) AFM image and $(b)$ cross-section of one of lines. $U=20 \mathrm{kV}$, $I=7 \mathrm{nA}, 60 \mathrm{~s}$ of exposure.
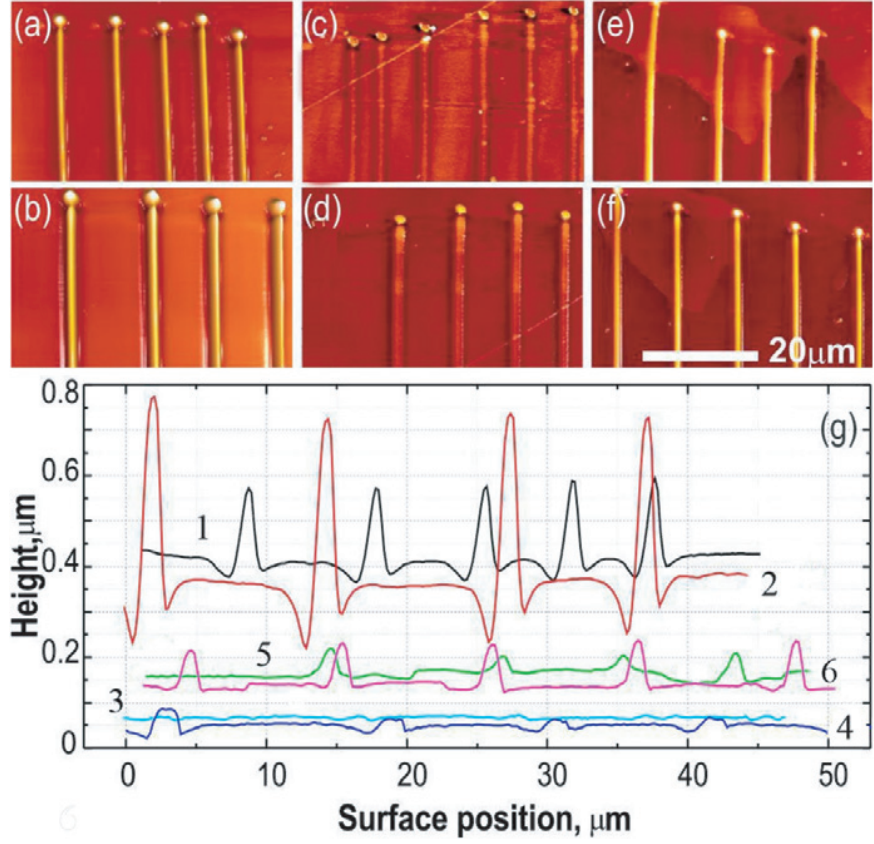

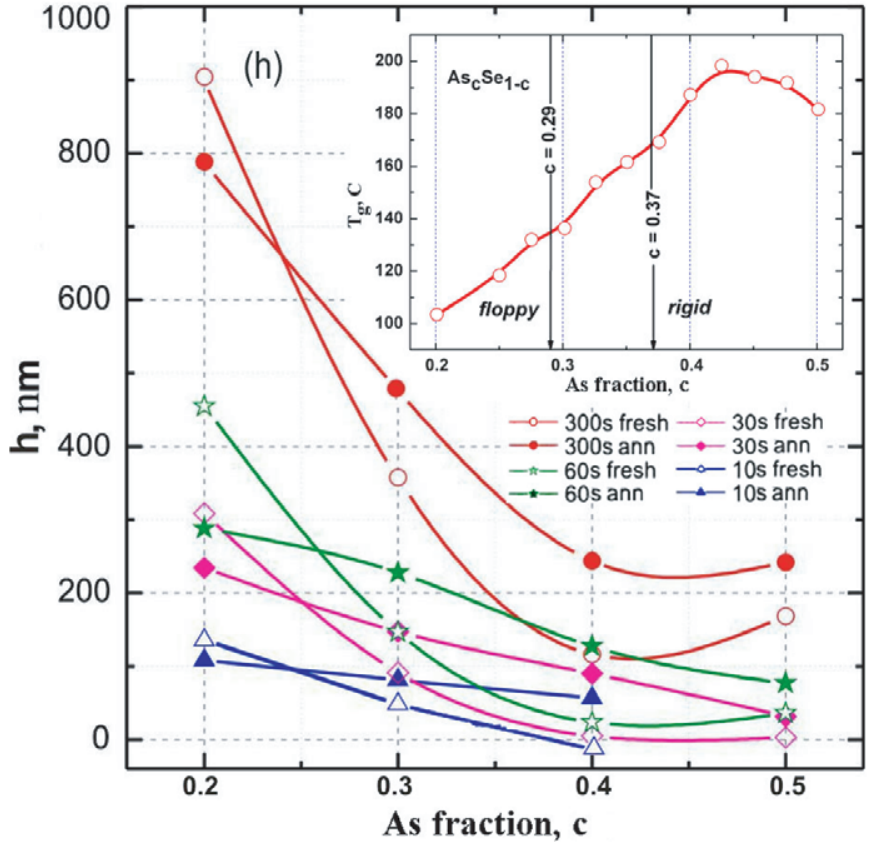

Figure 3. AFM images of e-beam lines obtained after 10 and $30 \mathrm{~s}$ exposure at $U=20 \mathrm{kV}$ for As-Se films of some compositions: $(a)$ and $(b)$ fresh $\mathrm{As}_{0.2} \mathrm{Se}_{0.8} ;(c)$ and $(d)$ fresh $\mathrm{As}_{0.4} \mathrm{Se}_{0.6} ;(e)$ and $(f)$ annealed $\mathrm{As}_{0.4} \mathrm{Se}_{0.6} ;(\mathrm{g})$ appropriate cross-sections: (1) and (2) fresh $\mathrm{As}_{0.2} \mathrm{Se}_{0.8} ;(3)$ and (4) fresh $\mathrm{As}_{0.4} \mathrm{Se}_{0.6} ;(5)$ and (6) annealed $\mathrm{As}_{0.4} \mathrm{Se}_{0.6}$. (h) Concentration dependence of the height of e-beam lines for $\mathrm{As}_{c} \mathrm{Se}_{1-c}$ films. The exposures are indicated directly in the image $(h)$. In insert: summary of results on $\operatorname{As}_{c} \operatorname{Se}_{1-c}$ bulk glasses showing $T_{\mathrm{g}}(c)$ variation. The data points are taken from [28].

figure 3( $\mathrm{g})$, curves 3 and 4. Similar behaviour was observed by other investigators earlier [18]. However, SRs are far more prominent in annealed films (figures 3, curves 5 and 6) especially under prolonged e-beam exposure, more than $60 \mathrm{~s}$ (see appropriate curves in figures $3(h)$ ).

Second, as it is shown in figure $3(h)$, alteration in e-beaminduced surface change occurs both in the fresh and annealed films. It is surprising that e-beam irradiation can provide more prominent SR formation in the annealed films compared to as-deposited films (except to $\mathrm{As}_{0.2} \mathrm{Se}_{0.8}$ composition, where the effects are comparable). The opposite situation usually takes place if we consider classical photostructural changes (e.g. photodarkening) induced by the band-gap light [4], i.e. annealed films are less sensitive to the photostimulated changes of their structure. It was considered that as-deposited $\mathrm{As}_{c} \mathrm{Se}_{1-c}$ thin films with $c \geqslant 0.3$ tended to have a molecular structure containing $\mathrm{As}_{4} \mathrm{Se}_{4}$ sub-units instead of continuous network structure of well-annealed films. It means that the structure of as-deposited thin films differs to some extent from that of well-annealed films and this is amenable to irreversible structural changes absent in annealed films due to structure polymerization in response to light [32] and possibly to ebeam irradiation [33]. This stands a possible reason of the different surface response in as-prepared and annealed $\mathrm{As}_{c} \mathrm{Se}_{1-c}$ films for $c \geqslant 0.3$. However, more detailed quantitative comparisons of the SR in different type of films under e-beam illumination remain to be studied and this topic is currently under investigation.

Third, figure 4(c) clearly shows that the height to the ridges difference and the depth of the depressions grow with the exposure without explicit saturation. The height of SR for $\mathrm{As}_{0.2} \mathrm{Se}_{0.8}$ composition reaches about $900 \mathrm{~nm}$ during $300 \mathrm{~s}$ 

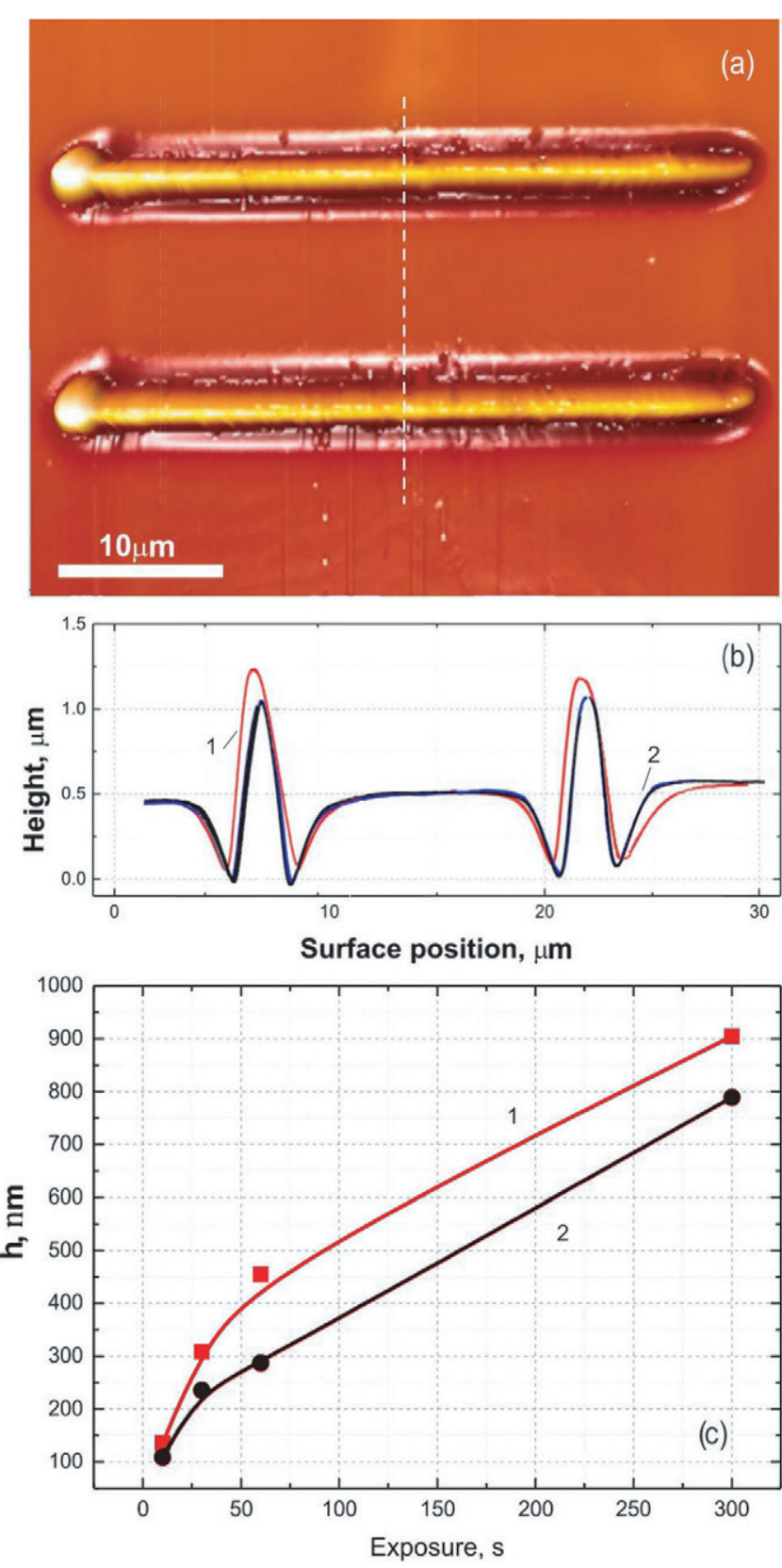

Figure 4. (a) AFM image of e-beam lines obtained after $300 \mathrm{~s}$ recording for fresh $\mathrm{As}_{0.2} \mathrm{Se}_{0.8}$ film; $(b)$ appropriate cross-sections; (c) the dependence of height of e-beam lines versus exposure for (curve 1) as-deposited and (curve 2) annealed film.

exposure, i.e. about $45 \%$ of initial film thickness. It is clear that ridge formation cannot be caused by a simple e-beam-induced expansion of the film material as discussed previously [20]; but it is caused by lateral mass transfer induced by e-beam, which produces micrometre-scale surface modifications and can be referred to as giant due to its prominence.

The time (exposure) dependence of the SR height consists of at least two stages, which occur with different rates and give different contributions in the profile variation, (see figure $4(c)$, curves 1 and 2). The first stage is detected immediately after beginning of irradiation and results in relatively small deformations, whereas the second stage is much slower, however, results in giant changes of the profile. The last changes are caused by lateral mass transport induced by ebeam, while the first step seems to be a simple volume expansion.

\section{Discussion}

The results obtained clearly indicate that the height of SR under e-beam exposure in $\mathrm{As}_{c} \mathrm{Se}_{1-c}$ thin films decrease with addition of As. The reliefs are stable for long periods of time and do not show a tendency to relax with time. Relief features observed at different composition may be considered in terms of structural connectivity and e-beam-induced volume diffusion. Few related aspects are analysed below.

\subsection{Effect of temperature}

The close parallels in the glass-transition temperature $T_{\mathrm{g}}(x)$ and e-beam-induced SR variation with composition, and in particular, the local minimum near $c=0.4$, corresponding to the stoichiometric composition $\mathrm{As}_{0.4} \mathrm{Se}_{0.6}$, may led to the idea that the heat generated by an e-beam during the thin film irradiation caused the temperature increase and the relief formation was governed by the temperature (see insert in figure $3(h)$ ). Notice that in such a case, the SR will be induced by mass transport due to the thermal diffusion of some kind of atoms due to the local temperature gradients. Although numerous estimation of temperature increase has been reported for a band-gap irradiation by light, the same procedure for e-beam irradiation has received less attention. From some speculations [19] based on the results of the paper [33], it is believed that the increase in temperature for e-beam irradiation spot is less than $10 \mathrm{~K}$.

To exclude the temperature effect of e-beam irradiation in our experiment, one can estimate the temperature increase as follows. With the electron current $I_{\mathrm{e}}=7 \mathrm{nA}$ and the acceleration voltage $U=20 \mathrm{kV}$, the power of the e-beam is of the order of $P=140 \mu \mathrm{W}$, and the fraction $f \approx 70 \%$ is converted into heat of the film. The increase in the film temperature can be estimated in assumption that the e-beam is at rest, the power $P$ is homogeneously dissipated inside of hemisphere of radius $R$ ( $R$ is the electron range [34]) and there is a steady-state temperature distribution outside the hemisphere, such as

$$
T(r)=T_{0}+\frac{T_{R}-T_{0}}{r} R .
$$

Here, $T_{0}$ is the room temperature and $T_{R}$ is the temperature at the boundary of the hemisphere (excitation volume). Then, from the heat balance equation

$$
f P=-\left.2 \pi R^{2} \kappa_{q} \frac{\mathrm{d} T}{\mathrm{~d} r}\right|_{r=R},
$$

we obtain

$$
\Delta T=T_{R}-T_{0}=\frac{f P}{2 \pi \kappa_{q} R} .
$$

Here, $\kappa_{q}$ is the heat conductivity of the film. With $R \approx 1.5 \mu \mathrm{m}$, $\kappa_{q} \approx 1.2 \mathrm{~W} \mathrm{~m}^{-1} \mathrm{~K}^{-1}$ and $f P=100 \mu \mathrm{W}$, we have $\Delta T \approx 9 \mathrm{~K}$. 
In fact, this value is decreased when the beam is scanned over a larger area [35]. Even for relatively low migration energies, $Q_{\mathrm{m}}$, of the film constituents under e-beam irradiation, which were estimated as about $0.1 \mathrm{eV}$ (see below), the temperature rise in $10 \mathrm{~K}$ near the room temperature can increase the jump probability only for several per cent. Thus, we can neglect influence of the temperature rise on the mass transport kinetics.

\subsection{Effect of structural connectivity and rigidity percolation transition}

More evident is the interconnection of the basic structure with the stimulated transformations: the As-Se system is almost the ideal for explanation how the glass network rigidity influences the investigated optical or e-beam recording processes, based on the directed mass transport effects. Both the light and e-beam excitations, which stimulate built-in gradients of excited electron-hole pairs, and non-equilibrium defects cause mass transport under the conditions of decreased viscosity or so-called photo plasticity: viscosity of the material under illumination lowers to $10^{12} \mathrm{P}[8,36]$. Also, according to the results presented in [28], rigidity percolation occurs near $\mathrm{As}_{0.28} \mathrm{Se}_{0.72}$ composition in As-Se system for bulk glasses, giving additional rise to the high flexibility of glass network and mobility of it constituents, atoms and floppy clusters under the driving forces of build in gradients of chemical potential, mechanical and electric fields. Concerning to the structure of As-Se glasses, we can note 'chains crossing model', which was recently proposed in the case of Se-rich compositions like $\mathrm{As}_{0.2} \mathrm{Se}_{0.8}$ and $\mathrm{As}_{0.3} \mathrm{Se}_{0.7}$ glasses [37]. Based on the elastic response of $\mathrm{ChG}$, their structural connectivity and rigidity reveals floppy, intermediate and stress-rigid phases [28]. It means that in the terms of topology with increasing As content, the glasses of As-Se system transit from being flexible (floppy) to over-coordinated stress-rigid but after passing some selforganized residual stress-free intermediate phase that starts near $\mathrm{As}_{0.28} \mathrm{Se}_{0.72}$ composition (at mean coordination number $r=2.29$ ). Near-absence of stress in the intermediate phase gives a possibility to large-scale response in the glass backbone under external excitation [30]. It causes giant SR formation when such glasses or appropriate amorphous films are exposed to e-beam. Contrary, after rigidity transition, when cross-links within a covalent network increases according to the compositional trends, the glasses transform to overconstrained rigid solid with structural backbone that contains locally stressed regions. Relaxation of these regions under interaction with e-beam, possibly, suppresses large atomic displacements leading to minimal surface deformation.

According to the model, the network of those samples built with comparable content of different structural fragments: (Se-Se-Se, As-Se-As and As-Se-Se). For example, the $\mathrm{As}_{0.2} \mathrm{Se}_{0.8}$ bulk glasses (that is characterized under-constrained network with mean coordination number $r=2.2$ ) contain $25 \%$ of $\mathrm{Se}$ atoms in $\mathrm{Se}-\mathrm{Se}-\mathrm{Se}$ chains and $75 \%$ of $\mathrm{Se}$ in As-Se-Se fragments, whereas all the As should be in $\mathrm{Se}-$ As $<(\mathrm{Se})_{2}$ structural units. Using this model for thin As-Se films, the mechanism of e-beam-induced mass transport could be associated with elementary diffusion act within $\operatorname{Se}_{n}(n \geqslant 3)$ chains (number $n$ of Se atoms in chain here is considered as a number of Se atoms between two As atoms). These elementary acts of diffusion that is starting in different parts of irradiated by e-beam place of the film attain a cooperative nature by initiating similar processes within the neighbouring structural units due to the inter-chain interactions and leads to the volume diffusion. It will be considered in detail in the next section.

At the same time, the stoichiometric and overstoichiometric compositions like $\mathrm{As}_{0.4} \mathrm{Se}_{0.6}$ and $\mathrm{As}_{0.5} \mathrm{Se}_{0.5}$ glasses formed by prevailed concentration of As-Se-As and contains in the latter case also considerable concentration of As-As- $(\mathrm{Se})_{2}$ structural fragments with homopolar As-As bonds. It is suggested that over-constrained networks with mean coordination number $r \geqslant 2.4$ that are characterized to be close to the stoichiometric composition $\left(\mathrm{As}_{0.4} \mathrm{Se}_{0.6}\right)$ and As-enriched one $\left(\mathrm{As}_{0.5} \mathrm{Se}_{0.5}\right)$ remain rigid despite e-beam excitation of bond constraints, therefore, precluding (depress) e-beam-induced structural rearrangements. The same consideration should be applicable to other ChG systems, where the special features of structural configurations should be taken into account. So, besides the structural, bonding constrains some other characteristics that should be taken into account when selecting the appropriate best material for ebeam prototyping of different elements.

\subsection{Shape of the profile and its concentration dependence}

Explanation of the shapes of the profiles and the concentration dependence of the patterning kinetics, which we observe in our experiments, requires a comprehensive analysis of the driving forces and the kinetic coefficients, which define ebeam-induced lateral mass transport. Previously [14, 27], we analysed patterning of $\mathrm{As}_{0.2} \mathrm{Se}_{0.8}$ films both under illumination by band-gap light and e-beam. We took into account variation of chemical potentials of the film constituents due to formation of radiation defects and a steady-state lateral distribution of electric potential, which is formed around the beam. Here, we propose another approach to estimate forming electric field and the kinetics of profile formation in the films of various concentrations.

The amorphous $\mathrm{As}_{c} \mathrm{Se}_{1-c}$ films are photoconductive p-type semiconductors with the atomic structure consisting mainly of chains with $P-C$ and partly $C-C$ bonds ( $P$ denotes pnictogen atom and $C$ denotes chalcogen). When the primary e-beam interacts with the sample, the electrons lose energy by repeated random scattering and absorption within a teardropshaped volume of the specimen is known as the interaction volume. The energy loss due to inelastic collisions is used, to a large extent, in the generation of electron-hole pairs. The generation rate can be calculated [39] as

$$
G_{0}=P / \chi E_{\mathrm{g}},
$$

where $P$ is the beam power, $E_{\mathrm{g}}$ is the band gap of the film material and $\chi$ represents an efficiency factor that includes losses due to backscattering and phonon interactions. As the film thickness, $H$, is almost equal to the electron range, $R$, the generation volume in a thin film is supposed to be a cylinder of radius $R$ and height $H$ ( $H$ is the film thickness), with constant 
generation rate inside. Then, the generation rate per unit of interaction volume is

$$
g_{0}=G_{0} / \pi R^{2} H .
$$

The generated free carriers move from the generated volume to all directions and change the local electrical properties of the material. As the electron and holes have different mobilities, their radial distribution results in formation of internal steadystate electric field, $E(r)$, which accelerates slower carriers and slows down faster carriers. In the $\mathrm{As}_{c} \mathrm{Se}_{1-c}$ films, mobility of holes exceeds mobility of electrons and thus the steadystate electric field should be directed towards the generated volume. Under irradiation by e-beam, scanning along a line in $y$-direction, one can consider lateral carrier distribution only in $x$-direction. Taking into account forming electric field, one can write the hole and electron currents as

$j_{p}=-D_{p} \frac{\mathrm{d} p}{\mathrm{~d} x}-p \frac{D_{p}}{k T} e E ; \quad j_{n}=-D_{n} \frac{\mathrm{d} p}{\mathrm{~d} x}+n \frac{D_{n}}{k T} e E$.

Here, $e$ is the electron charge, $n(x), p(x)$ and $D_{n}$ and $D_{p}$ are the local concentrations and the diffusion coefficients of electrons and holes, respectively, and $E(x)$ is the lateral electric field. The steady-state distribution corresponds to equality of electron and hole currents $\left(j_{n}=j_{p}\right)$; to satisfy the neutrality condition, one can suppose $n-n_{0}=p-p_{0}\left(n_{0}\right.$ and $p_{0}$ are the electron and hole concentrations without irradiation) and thus $\mathrm{d} p / \mathrm{d} x=\mathrm{d} n / \mathrm{d} x$. Then, the steady-state electric field is

$$
E=\frac{k T}{e} \cdot \frac{D_{p}-D_{n}}{p D_{p}+n D_{n}}
$$

and

$$
j_{p}=j_{n}=-D_{\mathrm{amb}} \frac{\mathrm{d} p}{\mathrm{~d} x} ; \quad D_{\mathrm{amb}}=\frac{(n+p) D_{n} D_{p}}{n D_{n}+p D_{p}} .
$$

Here, $D_{\text {amb }}$ is the ambipolar diffusion coefficient $[38,39]$. shape:

The distributions $p(x)$ and $n(x)$ have a Gaussian-like

$$
p-p_{0}=n-n_{0}=p_{\mathrm{m}} \cdot \exp \left(-x^{2} / l^{2}\right) .
$$

Here, $p_{\mathrm{m}}$ is maximum value of generated holes (and electrons), $l=\left(D_{\mathrm{amb}} \tau\right)^{1 / 2}, \tau$ is the lifetime of electrons and holes before recombination. It is worth noting that replacing the shape of the generation volume from cylinder to hemisphere of radius $R$ results in only marginal changes in the lateral distributions of $n(x), p(x)$ and $E(x)$. Estimates show that with $P=140 \mu \mathrm{W}$ and $R=2 \mu \mathrm{m}$ maximum values, $p_{\mathrm{m}}$ and $n_{\mathrm{m}}$ are of the order of $g_{0} \tau \approx 2 \times 10^{19} \mathrm{~cm}^{-3}$. A typical field distribution is shown in figure 5 for $l \approx 1 \mu \mathrm{m}$ [40]. It is negative with $x>0$ and positive at $x<0$ because the field is directed towards the interaction volume (around $x=0$ ).

After generation and redistribution, electrons and holes are localized around $P$ and $C$ atoms forming relatively stable configurations - valence alternation pairs - also called as selftrapped excitons (STE), which can be considered as radiation defects. Four main types of STE were found [41] to be relatively stable: $P_{2}^{-}-C_{3}^{+}, C_{1}^{-}-C_{3}^{+}, P_{2}^{-}-P_{4}^{+}$or $C_{1}^{-}-P_{4}^{+}$ (subscript number denotes the number of bonds and superscript

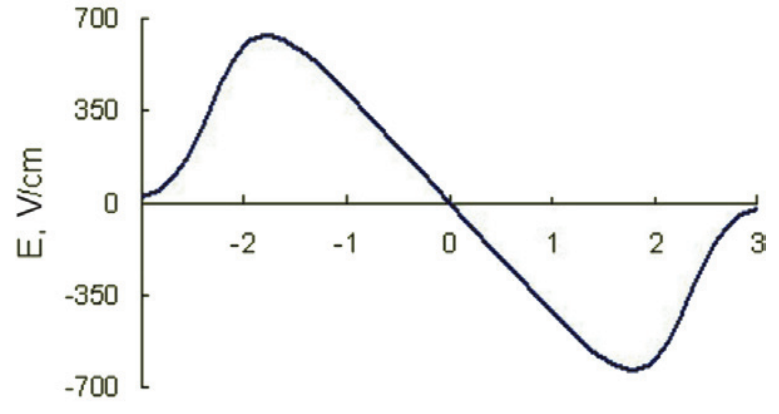

$x, \mu \mathrm{m}$

Figure 5. Typical distribution of lateral electric field $E(x)$ around the interaction volume $(x=0)$.

sign denotes the charge). Formation of these defects is accompanied by deformation and breakage of bonds and can accelerate atomic jumps compared to their thermal diffusion without irradiation [41]. This results in increase of the diffusion coefficients of both chalcogens and pnictides.

The calculations of the forming profiles were carried out in the following assumptions.

(i) The main mechanism of the mass transport is volume diffusion. This was assumed by analogy between e-beaminduced and light-induced mass transport in amorphous chalcogenide films. Recently, it was shown in [14] that the main mechanism of light-induced mass transport is volume diffusion.

(ii) Mobile species have an effective charge, $e$, positive or negative, since electrons and holes are localized near defects. Both $P$ and $C$ atoms can be charged either positively (such as $P_{4}^{+}$and $C_{3}^{+}$) or negatively $\left(P_{2}^{-}\right.$and $C_{1}^{-}$) depending on localization of electrons or holes around them.

(iii) Diffusion coefficients of the charged species are proportional to the local density of free volumes, whose concentration depends mainly on the local concentration of radiation defects, $n(x)$ and $p(x)$. As it follows from the atomic theory of diffusion [42], the diffusion coefficients can be defined as

$D_{k}=\frac{1}{2} \Gamma_{k} a^{2} ; \quad k=P_{4}^{+}, \quad P_{2}^{-}, \quad C_{3}^{+}, \quad C_{1}^{-}$.

Here, $a$ is the average length of elementary jump and $\Gamma_{k}$ is the jump frequency, which is defined by a probability of thermal activation near existing defect.

The frequencies $\Gamma_{k}$ can be estimated as

$$
\Gamma_{k} \approx n \bar{\omega} c_{k} \nu_{0} \exp \left(-Q_{\mathrm{m}}^{k} / k T\right)
$$

and the diffusion coefficients

$$
D_{k} \approx \frac{a^{2} \bar{\omega} c_{k}}{2} n v_{0} \exp \left(-Q_{\mathrm{m}}^{k} / k T\right) .
$$

Here, the product $n \bar{\omega} c_{k}$ defines a probability to meet a $k$-type of charged species near the free volume ( $\bar{\omega}=N^{-1}$ is the average atomic volume); $N$ is the 
total number of atoms per unit volume, $n \bar{\omega} \approx p \bar{\omega}$ is the dimensionless concentration of electrons and holes (which define concentration of the free volumes), $c_{k}$ is the atomic concentration of $k$-type of charged species, $v_{0}$ is the frequency of atomic oscillations and $Q_{\mathrm{m}}^{k}$ is the migration energies of $k$-type of species. The thermalinduced jumps are possible only due to existence of free volumes induced by electron irradiation and the jump probability is proportional to the defect concentration, $n \bar{\omega}$.

(iv) Each of the charged species is characterized by its diffusion coefficient, which depends on the number of bonds. Their migration energies are proportional to the average bonding energy, $u$, which is defined as $u=u_{P P} c^{2}+u_{C C}(1-c)^{2}+u_{P C} c(1-c)$, where $u_{P P}$, $u_{C C}$ and $u_{P C}$ are the energies of $P-P, C-C$ and $P-C$ bonds, respectively. This means that the migration energies of species $P_{4}^{+}$and $C_{3}^{+}$, which have four and three bonds, respectively, are higher than those for species $P_{2}^{-}$, which have two bonds, or for $C_{1}^{-}$, which have only one bond. This also means that the diffusion coefficients of negatively charged $P$ and $C$ atoms are higher compared to positively charged species. Unfortunately, we did not find any data about variation of $u$ with the rigidity of the material, and we took into account only variation of $u$ with the composition.

(v) Lateral diffusion flux is defined as a drift of the charged species in the lateral electric field

$$
J_{x}=-\frac{D^{-}}{k T} n e E-\frac{D^{+}}{k T} p e E,
$$

where $D^{ \pm}=D_{P}^{ \pm} c+D_{C}^{ \pm}(1-c), c$ is atomic fracture of $P$ atoms in the compound $\mathrm{As}_{c} \mathrm{Se}_{1-c}$ and $D_{P}^{ \pm}$and $D_{C}^{ \pm}$ are diffusion coefficients of $P$ and $C$ atoms with the localized holes or electrons. The positively and negatively charged $P$ and $C$ atoms drift in opposite directions. The drift, however, does not change electro neutrality because electrons and holes diffuse much faster than the charged species and ensure neutrality of the film. The normal rate of variation of the surface profile due to lateral mass transport is

$$
V_{n}=-\bar{\omega} \cdot H \cdot \mathrm{d} J_{x} / \mathrm{d} x .
$$

The height of the profile, $h$, varies as a result of two processes: radiation-induced volume dilatation caused by formation of radiation defects and redistribution of material due to lateral mass transport. Variation of the profile, $h(x, t)$, was calculated as

$$
h(x)=V_{n} t+H \cdot n \cdot \Delta \omega .
$$

Here, $t$ is the exposure time and $\Delta \omega$ is the difference between the free volume caused by formation of radiation defect near STE and the average atomic volume. The first term in equation (15) describes the contribution of the free volumes generated by formation of additional electrons and holes under e-beam irradiation.

In figure 6 , we present the profiles $h(x)$ calculated for various film compositions irradiation along a line. As fitting

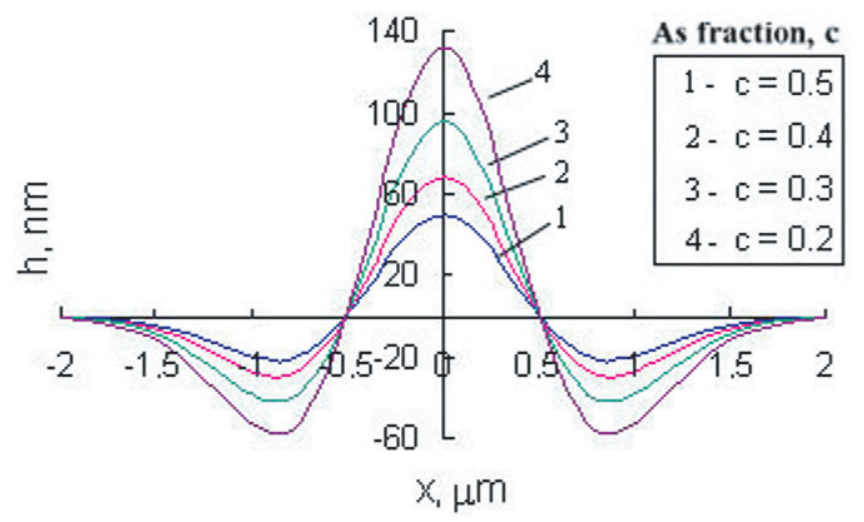

Figure 6. Calculated profiles produced by e-beam scanning along a line during $30 \mathrm{~s}$ in the films of various compositions. $P=140 \mu \mathrm{W}$.

parameters, we used efficiency factor $\chi$ and the migration energies, which define the effective diffusion coefficients $D^{-}$ and $D^{+}$corresponding to maximum carrier concentrations $n_{m}$ and $p_{m}($ at $x=0)$. Parameters used for the profile calculations are presented in table 1 .

In figure 7 , the height of the ridges, $h_{\mathrm{m}}$, calculated for a given exposure $(t=30 \mathrm{~s})$ is presented as function of the As concentration, $c$, in comparison with the experimental heights for fresh and annealed films.

The comparison shows rather good agreement between the calculated and experimental heights that confirms reasonability of the above assumptions. To get this agreement, we used $l=1 \mu \mathrm{m}, \chi=2, D^{-}=1.4 \times 10^{-9} \mathrm{~cm}^{2} \mathrm{~s}^{-1}$ and $D^{+}=0.1 D^{-}$. Some difference between the theory and experiment, especially at $c=0.2$, can be attributed to variation in configuration of radiation defects at different compositions.

\section{Conclusions}

It was established that the extent of e-beam-induced local surface deformations i.e. the height of the surface relief at the same expositions decreases with increasing As content in $\mathrm{As}_{c} \mathrm{Se}_{1-c}$ amorphous chalcogenide layers. For long-time exposure $(300 \mathrm{~s})$ in Se-enriched films ( $\mathrm{As}_{0.2} \mathrm{Se}_{0.8}$ composition), we obtained giant, up to the film thickness, surface relief.

It was established through AFM measurements that the time (exposure) dependence of the surface relief consists of at least two stages, which occur with different rates and give different contributions in the profile variation. The first stage is detected immediately after beginning of e-beam irradiation and may be connected with local volume change, which results in relatively small deformations, whereas the second stage is much slower, however, results in giant changes of the profile. These changes are caused by lateral mass transport induced by e-beam. The driving force of the mass transport is mainly defined by lateral steady-state electric field caused by steadystate distribution of electrons and holes generated by e-beam.

It may be concluded that both of the optical- and e-beamstimulated surface reliefs in As-Se system correlate with the rigidity percolation range and the maximum photoplasticity, which are not directly connected to the known photodarkening effect since it is minimal for those compositions. More 
Table 1. Migration energies, diffusion coefficients (at $x=0$ ) and efficiency factor used for calculations of surface profile variations. Coefficients $D^{-}$and $\chi$ were used as fitting parameters.

\begin{tabular}{llllllll}
\hline Atomic conc. $(c)$ & $Q_{C}^{-}(\mathrm{eV})$ & $Q_{C}^{+}(\mathrm{eV})$ & $Q_{P}^{-}(\mathrm{eV})$ & $Q_{P}^{+}(\mathrm{eV})$ & $D_{\max }^{+}\left(\mathrm{cm}^{2} \mathrm{~s}^{-1}\right)$ & $D_{\max }^{-}\left(\mathrm{cm}^{2} \mathrm{~s}^{-1}\right)$ & $\chi$ \\
\hline 0.2 & 0.095 & 0.285 & 0.190 & 0.380 & $5.4 \times 10^{-13}$ & $3.1 \times 10^{-8}$ & 2 \\
0.3 & 0.097 & 0.291 & 0.193 & 0.387 & $5.4 \times 10^{-13}$ & $2.2 \times 10^{-8}$ & 2 \\
0.4 & 0.097 & 0.292 & 0.195 & 0.390 & $2.8 \times 10^{-13}$ & $1.6 \times 10^{-8}$ & 2 \\
0.5 & 0.097 & 0.291 & 0.194 & 0.387 & $3.0 \times 10^{-13}$ & $1.15 \times 10^{-8}$ & 2 \\
\hline
\end{tabular}

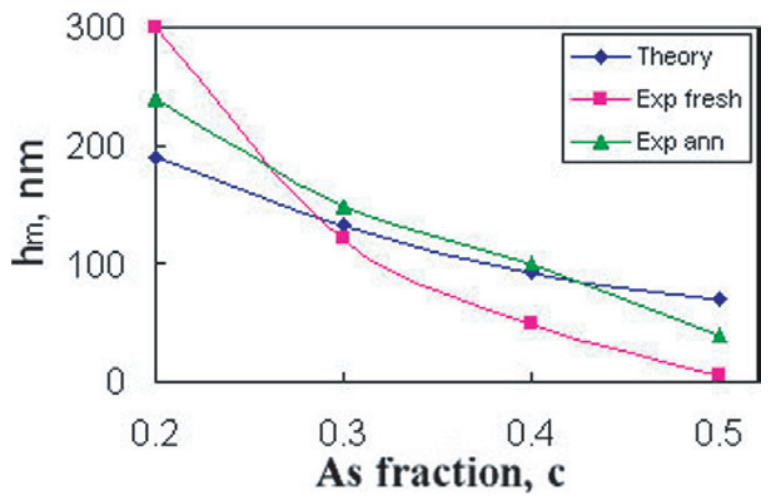

Figure 7. Comparison of the calculated ridge heights for various compositions with the experimental heights (see figure $3(h)$ ) for fresh and annealed films. Exposure time is $30 \mathrm{~s}$.

important is the defect structure, which enhances the diffusion processes in the gradients of excited electron-hole pairs and the localized electrons in the case of the e-beam excitation. The model of the process based on lateral mass transport due to volume diffusion is considered.

\section{Acknowledgments}

This work was supported by the TAMOP4.2.1./B-09/1/KONV2010-007 and 4.2.2.A-11/1/KONV-2012-0036 projects, which are co-financed by the European Union and European Social Fund.

\section{References}

[1] Tanaka K and Shimakawa K 2011 Amorphous Chalcogenide Semiconductors and Related Materials (Berlin: Springer)

[2] Bureau B et al 2004 J. Non-Cryst. Solids $\mathbf{3 4 5}$ and 346276

[3] Fritzsche H 2007 J. Phys. Chem. Solids 68878

[4] Kolobov A V 2003 Photo-Induced Metastability in Amorphous Semiconductors (Weinheim: Wiley-VCH)

[5] Nishihara H, Handa Y, Suhara T and Koyama J 1978 Appl. Opt. 171342

[6] Handa Y, Suhara T, Nishihara H and Koyama J 1979 Appl. Opt. 18248

[7] Ivan I, Veres M, Pocsik I and Kökényesi S 2004 Phys. Status Solidi a 2013193

[8] Yannopoulos S N and Trunov M L 2009 Phys. Status Solisi b 2461773

[9] Saliminia A, Galstian T V and Villeneuve A 2000 Phys. Rev. Lett. 854112

[10] Trunov M L, Lytvyn P M, Nagy P M and Dyachyns'ka O M 2010 Appl. Phys. Lett. 96111908

[11] Trunov M L, Lytvyn P M and Dyachyns'ka O M 2010 Appl. Phys. Lett. 97031905
[12] Trunov M L, Lytvyn P M, Yannopoulos S N, Szabo I A and Kökényesi S 2011 Appl. Phys. Lett. 99051906

[13] Kaganovskii Yu, Beke D L, Charnovych S, Kökényesi S and Trunov M L 2011 J. Appl. Phys. 110063502

[14] Kaganovskii Yu, Trunov M L, Beke D L and Kökényesi S 2012 Mater. Lett. 66159

[15] Kovalskiy A, Cech J, Tan C L, Heffner W R, Miller E Waits C M, Dubey M, Churaman W, Vlcek M and Jain H 2009 Proc. SPIE $\mathbf{7 2 7 3}$ 72734A1

[16] Kovalskiy A, Cech J, Vlcek M, Waits Ch M, Dubey M, Heffner W R and Jain H 2009 J. Micro/Nanolithogr. MEMS MOEMS 8/4 043012

[17] Nordman N and Salminen O 1996 Solid State Commun. 100241

[18] Nordman O, N Nordman N and Peyghambarian N 1998 J. Appl. Phys. 846055

[19] Tanaka K 1997 Appl. Phys. Lett. 70261

[20] Romero J S, Fitzgerald A G and Mietzsch K 2002 J. Appl. Phys. 919572

[21] Kovalskiy A, Neilson J R, Miller A C, Miller F C, Vlcek M and Jain H 2008 Thin Solid Films 5167511

[22] Liu W C, Hoffmann, Zhou W, Reano R M, Boolchand P and Sooryakumar R 2008 Appl. Phys. Lett. 93041107

[23] Hoffman G B, Zhou W, Sooryakumar R, Boolchand P and Reano R M 2009 J. Vac. Sci. Technol. B 272737

[24] Hoffman G B, Liu W-C, Zhou W, Sooryakumar R, Boolchand P and Reano R M. 2008 J. Vac. Sci. Technol. B 26278

[25] Takats V, Miller F, Jain H, Cserhati C and Kökényesi S 2009 Phys. Status Solidi c 6 S83

[26] Takats V, Nemec P, Miller A C, Jain H and Kökényesi S 2010 Opt. Mater. 32677

[27] Cserhati C, Charnovych S, Lytvyn P M, Trunov M L, Beke D L, Kaganovskii Yu and Kökényesi S 2012 Mater. Lett. 85113

[28] Georgiev D G, Micoulaut M and Boolchand P 2000 Phys Rev B 62 R9228

[29] Tanaka K 1989 Phys. Rev. B 391270

[30] Gump J, Finkler I, Xia H, Sooryakumar R, Bresser W J and Boolchand P 2004 Phys. Rev. Lett. 92245501

[31] Singh J and Shimakawa K 2003 Advances in Amorphous Semiconductors (London: Taylor and Francis)

[32] Neufville J P, Moss S C and Ovshinsky S R 1973-1974 J. Non-Cryst. Solids 13191

[33] Mee P B 1975 Thin Solid Films 26227

[34] Reimer L 1985 Scanning Electron Microscopy (Berlin: Springer)

[35] Pittaway L G 1964 Br. J. Appl. Phys. 15967

[36] Trunov M L 2008 J. Phys. D: Appl. Phys. 41074011

[37] Golovchak R, Kovalskiy A, Miller A C, Jain H and Shpotyuk O 2007 Phys. Rev. B 76125208

[38] Roosbroeck W 1953 Phys. Rev. B 91282

[39] Thomas Ch, Joachimsthaler I, R. Heiderhoff R and Balk L J 2004 J. Phys. D: Appl. Phys. 372785

[40] Regmi A, Ganjoo A, Zhao D, Jain H and Biaggio I 2012 Appl. Phys. Lett. 101061911

[41] Fritzsche H 1998 Semiconductors 32850

[42] Shewmon P G 1989 Diffusion in Solids 2nd edn (New York: Wiley) 


\section{QUERIES}

\section{Page 1}

AQ1

Please be aware that the colour figures in this article will only appear in colour in the web version. If you require colour in the printed journal and have not previously arranged it, please contact the Production Editor now.

Page 2

AQ2

Please provide the full form of 'ITO' in the sentence 'Amorphous films with....'

Page 2

AQ3

Please confirm the full form of 'EDAX' in the sentence 'The film thicknesses....'

\section{Page 2}

AQ4

Please confirm the full form of 'CFE' in the sentence 'Recording of lines...'.

\section{Page 9}

AQ5

Please check the details for any journal references that do not have a blue link as they may contain some incorrect information. Pale purple links are used for references to arXiv e-prints.

Page 9

AQ6

Please confirm whether the initials set for 'Ovshinsky S R' in ref. [32] are correct. 